\title{
Radiation of water waves by a heaving submerged horizontal disc
}

\author{
By P. A. MARTIN AND L. FARINA \\ Department of Mathematics, University of Manchester, Manchester M13 9PL, UK
}

(Received 30 July 1996 and in revised form 4 November 1996)

A thin rigid plate is submerged beneath the free surface of deep water. The plate performs small-amplitude oscillations. The problem of calculating the radiated waves can be reduced to solving a hypersingular boundary integral equation. In the special case of a horizontal circular plate, this equation can be reduced further to onedimensional Fredholm integral equations of the second kind. If the plate is heaving, the problem becomes axisymmetric, and the resulting integral equation has a very simple structure; it is a generalization of Love's integral equation for the electrostatic field of a parallel-plate capacitor. Numerical solutions of the new integral equation are presented. It is found that the added-mass coefficient becomes negative for a range of frequencies when the disc is sufficiently close to the free surface.

\section{Introduction}

Consider the radiation of small-amplitude time-harmonic surface water waves by an oscillating thin rigid plate, in three dimensions. The problem is to calculate the radiated waves and the hydrodynamic forces on the plate. If the plate lies in the free surface, such problems are known as dock problems. Circular docks have been studied by MacCamy (1961), Kim (1963), Miles \& Gilbert (1968), Garrett (1971), Miles $(1971,1987)$ and Maeda (1981). Dock problems are relatively simple because they can be reduced to a Fredholm integral equation of the second kind with a weakly singular kernel. In fact, the equation is a (direct) boundary integral equation for the velocity potential $\phi$ on the wetted surface of the plate. Maeda (1981) has also used this method for rectangular docks. Matched eigenfunction expansions have been used by Tanaka, Hamamoto \& Hashimoto (1981) to study the free vibrations of an elastic disc in the free surface of water of finite depth.

Suppose now that the plate is submerged. Then, we can reduce the problem to a hypersingular integral equation over the plate; the unknown is $[\phi]$, the discontinuity in the potential across the plate. Similar reductions have been made for two-dimensional problems by Parsons \& Martin (1992, 1994, 1995). Previous work on submerged plates in three dimensions is limited to the use of matched eigenfunction expansions in water of finite depth: this method was used by Yu \& Chwang (1993) for a horizontal circular plate and by Zhang \& Williams (1996) for a horizontal elliptical plate.

In this paper, we describe some of our work on the simplest situation, namely the heave (vertical) oscillations of a horizontal circular plate submerged beneath the free surface of deep water. This is an axisymmetric problem. We start from the governing hypersingular integral equation for $[\phi]$. We then explicitly invert the hypersingular part to obtain a Fredholm integral equation of the second kind. Finally, we introduce 
a new unknown function, leading to a very simple Fredholm integral equation of the second kind. This equation is easy to solve numerically (except when the disc is close to the free surface). It also has an elegant structure: for example, in the absence of waves, it reduces to Love's integral equation for the electrostatic field of a parallel-plate capacitor (Love 1949). The same equation (with a different forcing function) can be used to calculate the exciting force when an incident wavetrain is scattered by a fixed disc.

The motivation for the present work is twofold. First, we want some benchmark results which can be used for comparison with numerical solutions of the original hypersingular integral equation. Second, we are interested in the problem of small submergence, when (quasi-) resonant motions of the fluid above the disc are expected, by analogy with those found above a truncated vertical circular cylinder in water of constant finite depth by Longuet-Higgins (1967), Miles (1986) and others. That problem involves three dimensionless parameters whereas the problem treated here involves only two. Moreover, we have a rigorous reduction of the problem to a simple Fredholm integral equation of the second kind; this suggests the feasibility of a detailed asymptotic analysis for small depths of submergence.

\section{Boundary integral equations: submerged bodies}

A Cartesian coordinate system is chosen, in which $z$ is directed vertically downwards into the fluid, the undisturbed free surface lying at $z=0$. A body, with surface $S$, is completely submerged below the free surface of the fluid; $S$ is a smooth closed bounded surface. We consider small-amplitude time-harmonic motions. We use the assumptions of an inviscid incompressible fluid, and an irrotational motion, to allow the introduction of a velocity potential $\operatorname{Re}\left\{\phi(x, y, z) \mathrm{e}^{-\mathrm{i} \omega t}\right\}$, where $\omega$ is the frequency. The conditions to be satisfied by $\phi$ are Laplace's equation,

$$
\left(\frac{\partial^{2}}{\partial x^{2}}+\frac{\partial^{2}}{\partial y^{2}}+\frac{\partial^{2}}{\partial z^{2}}\right) \phi=0, \quad \text { in the fluid, } D,
$$

along with the free-surface condition

$$
K \phi+\frac{\partial \phi}{\partial z}=0 \quad \text { on } z=0,
$$

where $K=\omega^{2} / g$ and $g$ is the acceleration due to gravity. On the surface of the body, the normal velocity is prescribed, that is

$$
\frac{\partial \phi}{\partial n}=V \quad \text { on } S,
$$

where $V$ is a given function. In addition, $\phi$ must satisfy a radiation condition:

$$
r^{1 / 2}\left(\frac{\partial \phi}{\partial r}-\mathrm{i} K \phi\right) \rightarrow 0 \quad \text { as } r=\left(x^{2}+y^{2}\right)^{1 / 2} \rightarrow \infty .
$$

In what follows, we use capital letters $P, Q$ to denote points in the fluid, and lower-case letters $p, q$ to denote points on the submerged body.

Next, we reduce the boundary-value problem for $\phi$ to a boundary integral equation over $S$. To do this, we combine an appropriate fundamental solution with an application of Green's theorem. We use the fundamental solution corresponding to a three-dimensional wave source, defined by

$$
G(P, Q) \equiv G(x, y, z ; \xi, \eta, \zeta)=\left(R^{2}+(z-\zeta)^{2}\right)^{-1 / 2}+G_{1}(R, z+\zeta),
$$


where $R=\left((x-\xi)^{2}+(y-\eta)^{2}\right)^{1 / 2}$,

$$
G_{1}(R, z+\zeta)=\psi_{0}^{\infty} \mathrm{e}^{-k(z+\zeta)} J_{0}(k R) \frac{k+K}{k-K} \mathrm{~d} k
$$

and $J_{0}$ is a Bessel function. $G$ satisfies (2.1) and (2.2), and it has a simple singularity at $P=Q$ (behaving there as the inverse of the distance between $P$ and $Q$ ); the integration path in (2.6) is indented below the pole of the integrand at $k=K$ so that $G$ also satisfies the radiation condition. Note that $G_{1}(R, Z)$ is non-singular whenever $Z>0$.

Applying Green's theorem to $\phi(P)$ and $G(P, Q)$, we find that

$$
\phi(P)=\frac{1}{4 \pi} \int_{S}\left\{\phi(q) \frac{\partial G(P, q)}{\partial n_{q}}-G(P, q) \frac{\partial \phi}{\partial n_{q}}\right\} \mathrm{d} s_{q},
$$

where $P$ is any point in $D$ and $\partial / \partial n_{q}$ denotes normal differentiation at $q$ on $S$ in the direction from $S$ into $D$. This is the familiar integral representation for $\phi$ in the fluid in terms of $\phi$ and $\partial \phi / \partial n$ on $S$; the latter is known from the boundary condition (2.3), whence (2.7) becomes

$$
\phi(P)=\frac{1}{4 \pi} \int_{S}\left\{\phi(q) \frac{\partial G(P, q)}{\partial n_{q}}-G(P, q) V(q)\right\} \mathrm{d} s_{q} .
$$

Letting $P \rightarrow p$, a point on $S$, we obtain

$$
2 \pi \phi(p)-\int_{S} \phi(q) \frac{\partial G(p, q)}{\partial n_{q}} \mathrm{~d} s_{q}=-\int_{S} G(p, q) V(q) \mathrm{d} s_{q},
$$

which is a well-known boundary integral equation for $\phi$ on $S$. This equation is uniquely solvable for all values of $K$. Once solved, $\phi$ is given everywhere in the fluid by $(2.8)$.

\section{Boundary integral equations: plates}

Let the volume of the body in the previous section shrink, so that the body degenerates into a thin rigid plate. Thus, $S$ degenerates into $\Omega$, a finite bounded smooth open surface, with boundary $\partial \Omega$; we assume that $\partial \Omega$ (the plate's edge) is a smooth simple closed curve. $\Omega$ has two sides, $\Omega^{+}$and $\Omega^{-}$. The boundary-value problem for $\phi$ becomes: solve Laplace's equation (2.1) in $D$, subject to the free-surface condition (2.2), the radiation condition (2.4) and the boundary condition

$$
\frac{\partial \phi}{\partial n^{ \pm}}=V\left(p^{ \pm}\right) \quad \text { for } p^{ \pm} \text {on } \Omega^{ \pm},
$$

where $\partial / \partial n^{ \pm}$denote normal differentiation at a point on $\Omega^{ \pm}$in the direction from $\Omega^{ \pm}$ into $D$, and $p^{ \pm}$are corresponding points on $\Omega^{ \pm}$. Note that $\partial / \partial n^{+}=-\partial / \partial n^{-}$. In addition, we require that $\phi$ be bounded in the neighbourhood of $\partial \Omega$.

The velocity potential is discontinuous across $\Omega$ : define

$$
[\phi(p)]=\phi\left(p^{+}\right)-\phi\left(p^{-}\right) .
$$

Also, as the plate is rigid, we have $V\left(p^{+}\right)=-V\left(p^{-}\right)$. Hence, the integral representation $(2.8)$ reduces to

$$
\phi(P)=\frac{1}{4 \pi} \int_{\Omega}[\phi(q)] \frac{\partial G(P, q)}{\partial n_{q}^{+}} \mathrm{d} s_{q} .
$$


This equation states that $\phi(P)$ can be represented as a double-layer potential, that is as a distribution of normal dipoles over $\Omega$. Note that $V$ does not appear explicitly in (3.2).

To use the integral representation (3.2), we have to find $[\phi]$. If we adopt the standard approach, that is we let $P$ go to $p^{+}$and $p^{-}$in turn, and subtract the result, we obtain the nugatory result $[\phi]=[\phi]$. Instead, we impose the boundary condition (3.1) on $\Omega^{+}$, giving

$$
\frac{1}{4 \pi} \frac{\partial}{\partial n_{p}^{+}} \int_{\Omega}[\phi(q)] \frac{\partial G\left(p^{+}, q\right)}{\partial n_{q}^{+}} \mathrm{d} s_{q}=V\left(p^{+}\right), \quad p^{+} \in \Omega^{+} .
$$

A similar equation is obtained if we apply the boundary condition on $\Omega^{-}$. Equation (3.3) is an integro-differential equation for $[\phi(q)], q \in \Omega$. It is to be solved subject to the edge condition

$$
[\phi]=0 \quad \text { around the edge } \partial \Omega ;
$$

physically, because the plate is completely submerged, we require that the discontinuity in pressure across the plate tends to zero as we approach its edge.

It is tempting simply to take the normal derivative at $p^{+}$in (3.3) under the integral sign, but this leads to a non-integrable integrand. The conventional way of dealing with this difficulty is to regularize (3.3); various possibilities are described by Martin \& Rizzo (1989). Instead, we adopt a more direct approach. Thus, it can be proved that interchanging the order of integration and normal differentiation at $p^{+}$in (3.3) is legitimate, provided that the integral is then interpreted as a finite-part integral. By adopting this procedure, we find

$$
\frac{1}{4 \pi} \oint_{\Omega}[\phi(q)] \frac{\partial^{2}}{\partial n_{p}^{+} \partial n_{q}^{+}} G\left(p^{+}, q\right) \mathrm{d} s_{q}=V\left(p^{+}\right), \quad p^{+} \in \Omega^{+},
$$

which is to be solved for $[\phi]$, subject to (3.4). The cross on the integral sign indicates that it is to be interpreted as a two-dimensional finite-part integral; see Appendix A for further details.

The hydrodynamic force on the plate can be computed by integrating the pressure over both sides of $\Omega$ (Newman 1977). It is given by $\operatorname{Re}\left\{\boldsymbol{F} \mathrm{e}^{-\mathrm{i} \omega t}\right\}$, where

$$
\boldsymbol{F}=-\mathrm{i} \omega \rho \int_{\Omega}[\phi(q)] \boldsymbol{n}^{+}(q) \mathrm{d} s_{q},
$$

$\rho$ is the fluid's density and $\boldsymbol{n}^{+}$is the unit normal to $\Omega^{+}$pointing into the fluid. Thus, the force is given by an integral of $[\phi]$ over the plate.

\section{Submerged horizontal disc}

So far, we have not exploited the fact that $\Omega$ is a flat circular disc; indeed, the hypersingular integral equation (3.5) is valid when $\Omega$ is any smooth open bounded surface. We could solve the integral equation, numerically, using a boundary element method. Alternatively, if $\Omega$ is flat, we could use an appropriate expansion-collocation method (such methods are very effective for one-dimensional hypersingular integral equations). Indeed, one motivation for the present work is to validate such numerical methods.

We start by assuming that $\Omega$ is a circular disc of radius $a$. We can nondimensionalize using $a$; thus, without loss of generality, we take $a=1$. Next, assume that the disc is horizontal, with its centre at $(x, y, z)=(0,0, b / 2)$. We take $\Omega^{+}$ 
to be the lower side of the disc, so that $\partial / \partial n^{+}=\partial / \partial z$ evaluated on $z=b / 2$. Then (2.5) and (2.6) give

where

$$
\frac{\partial^{2} G}{\partial n_{p}^{+} \partial n_{q}^{+}}=\frac{1}{R^{3}}+M
$$

whence (3.5) becomes

$$
M=\psi_{0}^{\infty} \mathrm{e}^{-k b} J_{0}(k R) \frac{k^{2}(k+K)}{k-K} \mathrm{~d} k,
$$

$$
\frac{1}{4 \pi} \mho_{\Omega}[\phi(q)] \frac{\mathrm{d} \Omega}{R^{3}}+\frac{1}{4 \pi} \int_{\Omega}[\phi(q)] M(p, q) \mathrm{d} \Omega=V\left(p^{+}\right), \quad p^{+} \in \Omega^{+},
$$

where $\mathrm{d} \Omega$ is an element of area. This is a two-dimensional hypersingular integral equation. It is valid when $\Omega$ is any flat horizontal plate (not necessarily circular). Next, we shall reduce (4.1) to a set of uncoupled one-dimensional equations.

\section{One-dimensional equations}

Introduce plane polar coordinates $r$ and $\theta$ for the point $p=(x, y)$ on the disc; thus $x=r \cos \theta$ and $y=r \sin \theta$, so that $\Omega=\{(r, \theta): 0 \leqslant r<1,-\pi \leqslant \theta<\pi\}$. Similarly, for $q=(\xi, \eta)$, let $\xi=\rho \cos \varphi$ and $\eta=\rho \sin \varphi$ whence $\mathrm{d} \Omega=\rho \mathrm{d} \rho \mathrm{d} \varphi$. We now expand all quantities as Fourier series in the angular variables. Thus, suppose that

$$
V(r, \theta)=\sum V_{n}(r) \cos n \theta,
$$

where, in this section, all summations are from $n=0$ to $n=\infty$. Using

$$
J_{0}(k R)=\sum \epsilon_{n} J_{n}(k r) J_{n}(k \rho) \cos n(\theta-\varphi),
$$

where $\epsilon_{0}=1$ and $\epsilon_{n}=2$ for $n>1$, we obtain

$$
M(r, \theta ; \rho, \varphi)=\sum \epsilon_{n} M_{n}(r, \rho) \cos n(\theta-\varphi),
$$

where

$$
M_{n}(r, \rho)=\psi_{0}^{\infty} \mathrm{e}^{-k b} J_{n}(k r) J_{n}(k \rho) \frac{k^{2}(k+K)}{k-K} \mathrm{~d} k .
$$

Then, it follows from (4.1) that $[\phi]$ has a similar expansion,

$$
[\phi(r, \theta)]=\sum w_{n}(r) \cos n \theta,
$$

where $w_{n}$ satisfies a one-dimensional integral equation. To see this, we write (4.1) as

$$
\frac{1}{4 \pi} \nprec_{\Omega}[\phi] \frac{\mathrm{d} \Omega}{R^{3}}=f(p), \quad p \in \Omega,
$$

where

$$
f(r, \theta)=V(r, \theta)-\frac{1}{4 \pi} \int_{\Omega}[\phi(q)] M(p, q) \mathrm{d} \Omega .
$$

Substituting the various Fourier series, we obtain $f(r, \theta)=\sum f_{n}(r) \cos n \theta$, where

$$
f_{n}(r)=V_{n}(r)-\frac{1}{2} \int_{0}^{1} w_{n}(\rho) M_{n}(r, \rho) \rho \mathrm{d} \rho .
$$


Now, (5.4) arises in classical potential theory. For example, the problem of the flow of an ideal fluid past a thin rigid circular disc can be reduced to (5.4), wherein $f$ is known in terms of the ambient flow. Such problems can be solved exactly, usually by integral-transform techniques and dual integral equations (Sneddon 1966). Thus, $w_{n}$ and $f_{n}$ are related by the explicit formula

$$
w_{n}(r)=-\frac{4}{\pi} r^{n} \int_{r}^{1} \frac{1}{t^{2 n}\left(t^{2}-r^{2}\right)^{1 / 2}} \int_{0}^{t} \frac{s^{n+1} f_{n}(s)}{\left(t^{2}-s^{2}\right)^{1 / 2}} \mathrm{~d} s \mathrm{~d} t, \quad n=0,1,2, \ldots ;
$$

Guidera \& Lardner (1975) give a clear derivation of this result.

Substituting for $f_{n}$ from (5.5) into (5.6), we find that

$$
w_{n}(r)=w_{n}^{\infty}(r)+\int_{0}^{1} w_{n}(\rho) L_{n}(r, \rho) \mathrm{d} \rho, \quad 0 \leqslant r<1,
$$

where

$$
w_{n}^{\infty}(r)=-\frac{4}{\pi} r^{n} \int_{r}^{1} \frac{1}{t^{2 n}\left(t^{2}-r^{2}\right)^{1 / 2}} \int_{0}^{t} \frac{s^{n+1} V_{n}(s)}{\left(t^{2}-s^{2}\right)^{1 / 2}} \mathrm{~d} s \mathrm{~d} t
$$

and

$$
L_{n}(r, \rho)=\frac{2}{\pi} \rho r^{n} \int_{r}^{1} \frac{1}{t^{2 n}\left(t^{2}-r^{2}\right)^{1 / 2}} \int_{0}^{t} \frac{s^{n+1}}{\left(t^{2}-s^{2}\right)^{1 / 2}} M_{n}(s, \rho) \mathrm{d} s \mathrm{~d} t .
$$

Equation (5.7) is a Fredholm integral equation of the second kind for $w_{n} ; w_{n}^{\infty}$ is known: it gives the solution for $w_{n}$ if the forcing $V$ was applied to the disc in an unbounded ideal fluid, so that all the free-surface effects are included in the continuous kernel $L_{n}$. However, the integral equation is complicated; a much simpler equation is obtained next.

\section{Simpler one-dimensional equations}

Examination of (5.6), (5.8) and (5.9) suggests that a simpler integral equation can be obtained by introducing a new unknown function, $\psi_{n}$, related to $w_{n}$ by

$$
w_{n}(r)=D_{n} r^{n} \int_{r}^{1} \frac{\psi_{n}(t) \mathrm{d} t}{t^{n}\left(t^{2}-r^{2}\right)^{1 / 2}},
$$

where $D_{n}$ is a normalizing factor at our disposal. Comparison with (5.6) gives

$$
\psi_{n}(t)=\psi_{n}^{\infty}(t)+\frac{2}{\pi} t^{-n} \int_{0}^{t} \frac{s^{n+1} F_{n}(s)}{\left(t^{2}-s^{2}\right)^{1 / 2}} \mathrm{~d} s,
$$

where

$$
\begin{aligned}
\psi_{n}^{\infty}(t) & =-\frac{4}{\pi D_{n}} t^{-n} \int_{0}^{t} \frac{s^{n+1} V_{n}(s)}{\left(t^{2}-s^{2}\right)^{1 / 2}} \mathrm{~d} s, \\
F_{n}(s) & =\frac{1}{D_{n}} \int_{0}^{1} w_{n}(\rho) M_{n}(s, \rho) \rho \mathrm{d} \rho \\
& =\int_{0}^{1} \rho^{n+1} M_{n}(s, \rho) \int_{\rho}^{1} \frac{\psi_{n}(t) \mathrm{d} t}{t^{n}\left(t^{2}-\rho^{2}\right)^{1 / 2}} \mathrm{~d} \rho \\
& =\int_{0}^{1} \frac{\psi_{n}(t)}{t^{n}} \int_{0}^{t} \frac{\rho^{n+1}}{\left(t^{2}-\rho^{2}\right)^{1 / 2}} M_{n}(s, \rho) \mathrm{d} \rho \mathrm{d} t .
\end{aligned}
$$


Now, given the formula for $M_{n},(5.2)$, we can evaluate the integral over $\rho$ in (6.4) and the integral over $s$ in (6.2) using

$$
\frac{1}{y^{n}} \int_{0}^{y} \frac{\rho^{n+1} J_{n}(k \rho)}{\left(y^{2}-\rho^{2}\right)^{1 / 2}} \mathrm{~d} \rho=y j_{n}(k y),
$$

where $j_{n}(z)=\left(\frac{1}{2} \pi / z\right)^{1 / 2} J_{n+1 / 2}(z)$ is a spherical Bessel function. The result is that (6.2) reduces to

$$
\psi_{n}(x)-\int_{0}^{1} \psi_{n}(y) N_{n}(x, y) \mathrm{d} y=\psi_{n}^{\infty}(x), \quad 0 \leqslant x \leqslant 1,
$$

where

$$
N_{n}(x, y)=\frac{2}{\pi} x y \psi_{0}^{\infty} \mathrm{e}^{-k b} j_{n}(k x) j_{n}(k y) \frac{k^{2}(k+K)}{k-K} \mathrm{~d} k
$$

is a symmetric continuous kernel.

The idea of replacing $w_{n}$ by $\psi_{n}$ has been used previously by Martin \& Wickham (1983) for another problem involving a circular disc, namely the diffraction of elastic waves by a penny-shaped crack.

\section{The heaving disc}

Let us now make the simplest choice for the forcing $V$, namely

$$
V(r, \theta)=1 \text {. }
$$

This corresponds to heave (vertical) oscillations of the plate. It follows that the solution is axisymmetric: $[\phi]=w_{0}$. We choose

$$
D_{0}=-4 / \pi \quad \text { whence } \quad \psi_{0}^{\infty}(x)=x .
$$

Thus (writing $\psi \equiv \psi_{0}$ ), we have

$$
w_{0}(r)=-\frac{4}{\pi} \int_{r}^{1} \frac{\psi(t) \mathrm{d} t}{\left(t^{2}-r^{2}\right)^{1 / 2}}
$$

where $\psi$ solves

$$
\psi(x)-\int_{0}^{1} \psi(y) N_{0}(x, y) \mathrm{d} y=x, \quad 0 \leqslant x \leqslant 1 .
$$

This integral equation can be solved numerically by a straightforward method: the kernel is symmetric and continuous. Difficulties are expected only when $b$ is small, that is when the disc is close to the free surface.

Further simplifications are possible. Thus, as $j_{0}(z)=z^{-1} \sin z$, the kernel simplifies to

$$
\begin{aligned}
N_{0}(x, y) & =\frac{2}{\pi} \int_{0}^{\infty} \mathrm{e}^{-k b} \sin k x \sin k y \frac{k+K}{k-K} \mathrm{~d} k \\
& =L(x-y)-L(x+y)
\end{aligned}
$$

where

$$
L(X)=\frac{1}{\pi} \psi_{0}^{\infty} \mathrm{e}^{-k b} \cos k X \frac{k+K}{k-K} \mathrm{~d} k
$$

is an even function of $X$. Note that $N_{0}(x, y)$ is an odd function of $x: N_{0}(-x, y)=$ $-N_{0}(x, y)$. As the right-hand side of (7.2) is odd, the integral equation implies that 
$\psi(x)$ can be extended as an odd function of $x$. These properties allow us to write the integral equation as an equation over the interval $-1 \leqslant x \leqslant 1$; the result is

$$
\psi(x)-\int_{-1}^{1} \psi(y) L(x-y) \mathrm{d} y=x, \quad-1 \leqslant x \leqslant 1 .
$$

This is a Fredholm integral equation of the second kind with a smooth difference kernel $L ; \psi$ is the continuous odd solution of this equation.

We can separate the kernel into two parts, using

$$
\frac{k+K}{k-K}=1+\frac{2 K}{k-K} \text {. }
$$

This gives

$$
L(X)=L_{0}(X)+L_{1}(X)
$$

where

$$
L_{0}(X)=(b / \pi)\left(b^{2}+X^{2}\right)^{-1}, \quad L_{1}(X)=(2 K / \pi) \Phi_{0}(X, b)
$$

and

$$
\Phi_{0}(X, Y)=\psi_{0}^{\infty} \mathrm{e}^{-k Y} \cos k X \frac{\mathrm{d} k}{k-K}
$$

is a two-dimensional wave-source potential. $\Phi_{0}$ can be computed conveniently using an expansion derived by Yu \& Ursell (1961):

$$
\begin{aligned}
\Phi_{0}(X, Y)= & -\mathrm{e}^{-K Y}\{(\log K S-\mathrm{i} \pi+\gamma) \cos K X+\beta \sin K X\} \\
& +\sum_{m=1}^{\infty} \frac{(-K S)^{m}}{m !}\left(\frac{1}{1}+\frac{1}{2}+\cdots+\frac{1}{m}\right) \cos m \beta,
\end{aligned}
$$

where $S$ and $\beta$ are defined by $X=S \sin \beta$ and $Y=S \cos \beta$, and $\gamma=0.5772 \ldots$ is Euler's constant.

We note that $L_{0}$ is wave-free: it corresponds to replacing the free surface by a rigid lid $(K=0)$; see $\$ 8.2$ below. All wave effects are included in $L_{1}$.

Written explicitly, (7.4) becomes

$$
\psi(x)-\frac{b}{\pi} \int_{-1}^{1} \frac{\psi(y)}{b^{2}+(x-y)^{2}} \mathrm{~d} y-\frac{2 K}{\pi} \int_{-1}^{1} \psi(y) \Phi_{0}(x-y, b) \mathrm{d} y=x, \quad-1 \leqslant x \leqslant 1 .
$$

This is the final form of our equation for $\psi$. The solution depends on the wavenumber $K$ and the submergence $b$ (recall that the plate has radius 1 and is submerged at a distance $b / 2$ below the mean free surface); we write $\psi(x)=\psi(x ; K, b)$ when we wish to emphasize this dependence.

\subsection{Hydrodynamic force}

The hydrodynamic force on the heaving disc is given by (3.6). Suppose that the disc's centre is at $z=\frac{1}{2} b-(\mathscr{U} / \omega) \cos \omega t$, where $\mathscr{U}$ is a constant. Then, the vertical hydrodynamic force on the disc, $F$, can be expressed as

$$
F=\rho a^{3} \mathscr{U} \omega\{\mathscr{A} \cos \omega t+\mathscr{B} \sin \omega t\},
$$

where $a$ is the radius of the disc, and $\mathscr{A}$ and $\mathscr{B}$ are the dimensionless added-mass 
and damping coefficients, respectively. We find that

$$
\begin{aligned}
\mathscr{A}(K, b)+\mathrm{i} \mathscr{B}(K, b) & =-\int_{\text {unit disc }}[\phi] \mathrm{d} s=-2 \pi \int_{0}^{1} w_{0}(r) r \mathrm{~d} r \\
& =8 \int_{0}^{1} \psi(x) x \mathrm{~d} x,
\end{aligned}
$$

where we have used (7.1). Thus, the added mass and damping are easily computed in terms of the solution to (7.6).

Comparing the right-hand sides of (7.6) and (7.7) suggests multiplying (7.6) by the complex conjugate of $\psi$, and integrating over $x$. Taking the imaginary part of the result gives

$$
\mathscr{B}(K, b)=8 K \mathrm{e}^{-K b}\left|\int_{-1}^{1} \psi(x) \sin K x \mathrm{~d} x\right|^{2},
$$

which shows that the damping cannot be negative.

\section{Special cases}

Let us consider some special cases of the integral equation (7.6).

8.1. Deep submergence: $b \rightarrow \infty$

When $b \rightarrow \infty$, the kernel $L \rightarrow 0$, whence $\psi(x ; K, \infty)=x$. From (7.7), we find that

$$
\mathscr{A}=\frac{8}{3} \quad \text { and } \quad \mathscr{B}=0 \quad(b \rightarrow \infty) .
$$

Physically, this corresponds to a single disc oscillating in an unbounded fluid. The result (8.1) is well known and can be found in Lamb's book (1932, §102).

$$
\text { 8.2. } K=0 \text { : Love's equation }
$$

When $K=0$, the second integral term $\left(L_{1}\right)$ is absent. Thus, if we write $f(x)=$ $\psi(x ; 0, b)$, we find that

$$
f(x)-\frac{b}{\pi} \int_{-1}^{1} \frac{f(y)}{b^{2}+(x-y)^{2}} \mathrm{~d} y=x, \quad-1 \leqslant x \leqslant 1 .
$$

This is a well-studied equation, known as Love's equation (Love 1949). (Actually, Love's original equation has 1 rather than $x$ on the right-hand side.) It arises in the electrostatic problem of a circular plate condenser. This Dirichlet problem $(\phi$ prescribed on each disc) is discussed at length by Sneddon (1966, §8.1). In our case, we can see that setting $K=0$ in the free-surface condition (2.2) is equivalent to having a pair of identical coaxial discs in an unbounded perfect fluid, with $\partial \phi / \partial z=+1$ on one disc and $\partial \phi / \partial z=-1$ on the other; $b$ is the separation between the discs. We are not aware of a direct derivation of (8.2) for this potential problem, although it can be obtained from a paper by Collins (1961) on acoustic scattering by two coaxial rigid discs.

Despite its apparent simplicity, no closed-form solution of Love's equation is known. Such a solution was claimed by Atkinson, Young \& Brezovich (1983), but the claim was subsequently shown to be false by Hughes (1984) and by Love (1990) himself. 


$$
\text { 8.3. } K=\infty
$$

When $K \rightarrow \infty$, the split (7.5) is inappropriate; from (7.3), the limiting kernel is found to be

$$
L(X)=-b\left(b^{2}+X^{2}\right)^{-1} .
$$

Thus, writing $g(x)=\psi(x ; \infty, b)$, we find that $g$ satisfies

$$
g(x)+\frac{b}{\pi} \int_{-1}^{1} \frac{g(y)}{b^{2}+(x-y)^{2}} \mathrm{~d} y=x, \quad-1 \leqslant x \leqslant 1 .
$$

This equation differs from (8.2) by the sign of the integral term.

\section{Numerical results}

To solve the integral equation (7.6) numerically, for general values of $K$ and $b$, we use a Nyström method with the Gauss-Legendre quadrature rule. (Recall that the kernel is continuous.) Thus, we solve a linear system

$$
\sum_{j=1}^{N} A_{i j}(K, b) \psi_{j}=x_{i}, \quad i=1,2, \ldots, N,
$$

where $x_{i}$ is a quadrature node, $\psi_{i}=\psi\left(x_{i}\right)$,

$$
A_{i j}=\delta_{i j}-\frac{w_{j}}{\pi}\left\{\frac{b}{b^{2}+\left(x_{i}-x_{j}\right)^{2}}+2 K \Phi_{0}\left(x_{i}-x_{j}, b\right)\right\}
$$

and $w_{j}$ is a quadrature weight. We checked for convergence using various values of $N$; for small $b$, we found that $N=60$ gave good results, with smaller $N$ required for larger $b$. Equation (7.7) can be used to compute the added-mass and damping coefficients; thus

$$
\mathscr{A}+\mathrm{i} \mathscr{B} \approx 4 \sum_{j=1}^{N} w_{j} x_{j} \psi_{j} .
$$

Figure 1 shows the added-mass coefficient $\mathscr{A}$ as a function of $K a$, for three depths of submergence $b / 2$. We note the occurrence of negative added mass for sufficiently small $b$. Moreover, we notice increasingly sharp maxima and minima as $b \rightarrow 0$.

In figure 2 we see that these features are accompanied by a large peak in the damping coefficient $\mathscr{B}$. This confirms a result of McIver \& Evans (1984) deduced from the Kramers-Kronig relations. We can also observe that the height of the peak in the damping coefficient is almost exactly the same as the total height of the corresponding spikes in the added-mass coefficient. Furthermore, the peak of the damping is very close to a zero of the added mass. These properties were observed previously by Linton \& Evans $(1992,1993)$ in their studies of radiation by submerged bottom-mounted vertical circular cylinders in channels.

The formation of additional minor spikes in the added-mass coefficient should be noted also. These spikes are more evident for smaller values of $b$; see figure 3 .

The spikes in the graphs of the hydrodynamic coefficients occur at frequencies called resonant frequencies. They correspond to singularities of the system matrix $\boldsymbol{A}(K, b)$ (with entries $A_{i j}$ ) as a function of complex $K$, for fixed $b$. These values of $K$ are called scattering frequencies; for more information, see Hazard \& Lenoir (1993), Alves \& Ha Duong (1995) and Poisson (1995). Scattering frequencies are poles in 


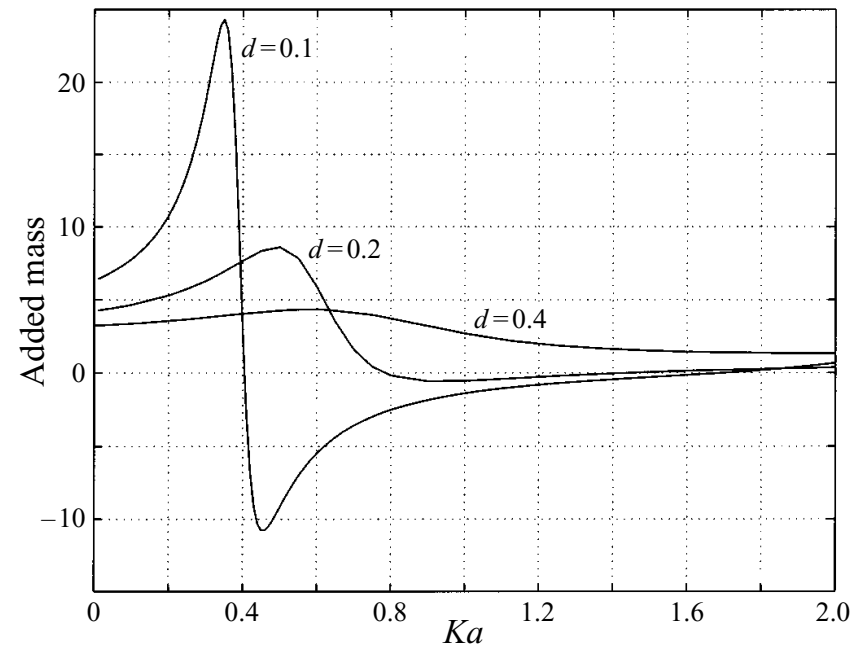

Figure 1. Added-mass coefficient $\mathscr{A}$ as a function of $K a$ for three values of $d$, where $d=b / 2$ is the submergence of the disc.

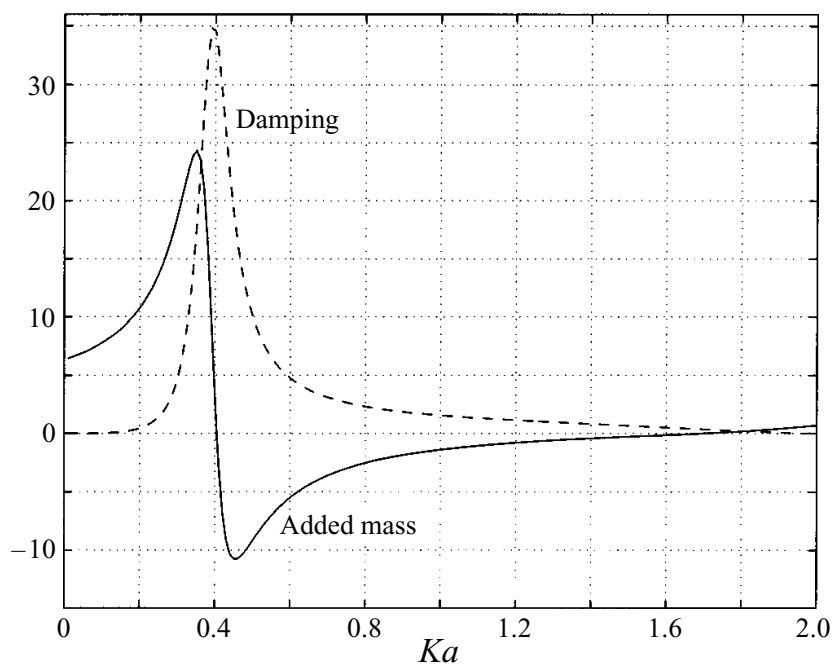

FIGURE 2. Added-mass and damping coefficients as a function of $K a$ for $b=0.2$.

the complex $K$-plane; they lie in $\operatorname{Im}(K)<0$. Numerically, they can be located by searching for zeros of $\operatorname{det} \boldsymbol{A}$ (Farina 1996).

For small $b$, it is natural to compare the hydrodynamic coefficients $\mathscr{A}$ and $\mathscr{B}$ with those for the dock problem $(b=0), \mathscr{A}_{d}$ and $\mathscr{B}_{d}$, respectively. In figure 4 , we compare the damping coefficients, $\mathscr{B}$ and $\mathscr{B}_{d}$, for $b=0.02$. We see that, apart from the spikes, the underlying trend of $\mathscr{B}$ is very close to $\mathscr{B}_{d}$, especially for larger values of $K a$. However, the same cannot be said of the added-mass coefficients; these are shown in figure 5 , again for $b=0.02$. In fact, we find numerically that, apart from the spikes, it is $\left|\mathscr{A}-\mathscr{A}_{d}+\pi /(K a)\right|$ which is small, especially for larger $K a$. This can be explained as follows. For small $b / a$, the potential beneath the submerged disc and the dock are the same. However, the potential in the thin layer above the submerged disc is significant; there, the fluid is in a simple state of uniform vertical 


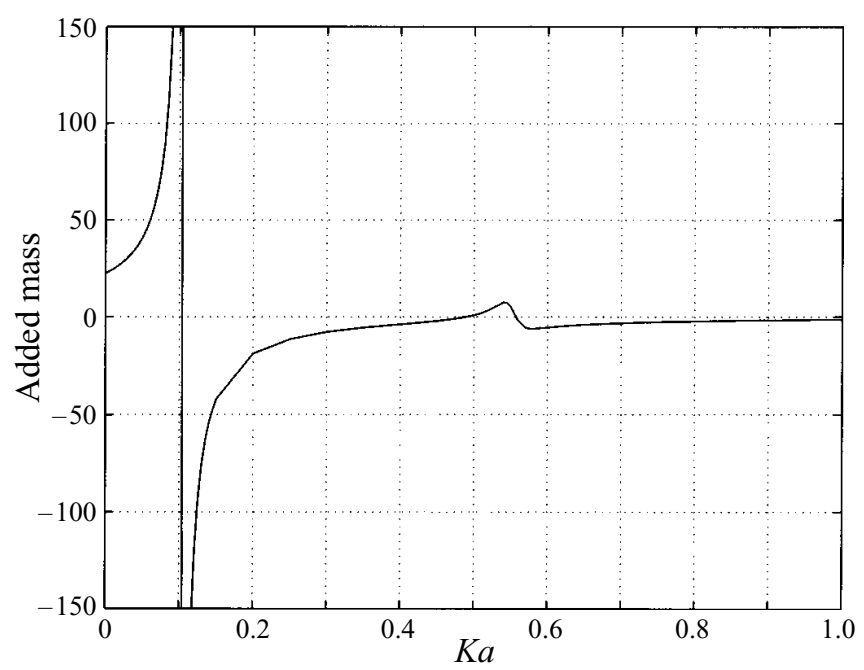

Figure 3. Added-mass coefficient $\mathscr{A}$ as a function of $K a$ for $b=0.04 . \mathscr{A}$ is of order $10^{3}$ at its extrema near $K a=0.1$.

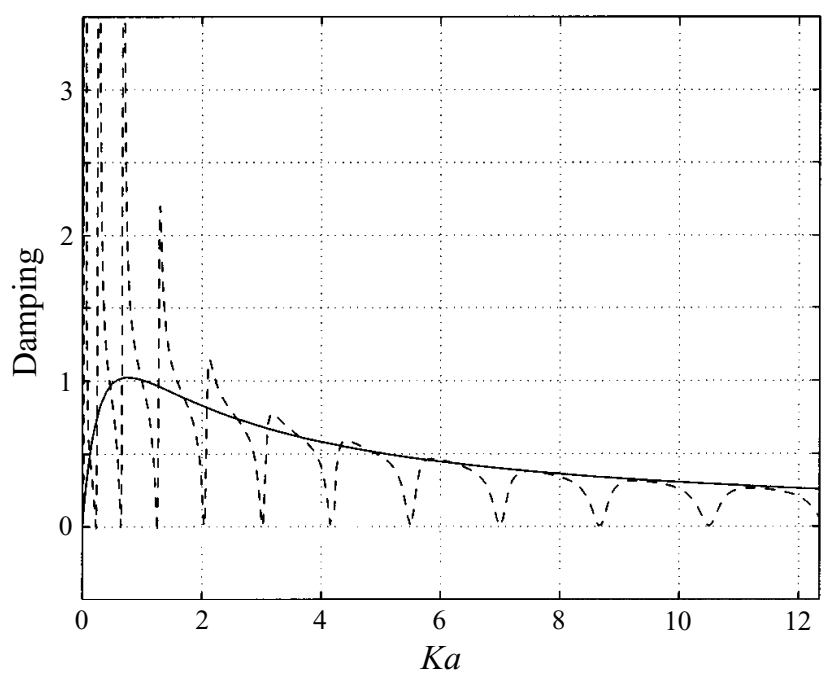

FIGURE 4. The damping coefficient for the submerged disc at $b=0.02$ (dashed curve) compared with the damping coefficient for the dock ( $b=0$; solid curve).

oscillation in phase with the disc, with potential $z-1 / K$ (see Newman, Sortland \& Vinje 1984). Thus, $[\phi] \simeq \phi_{d}+1 / K$, where $\phi_{d}$ is the potential on the dock. Hence, $\mathscr{A}+\mathrm{i} \mathscr{B} \simeq \mathscr{A}_{d}+\mathrm{i} \mathscr{B}_{d}-\pi /(K a)$, in agreement with the numerical results.

\section{Discussion}

The numerical results are especially interesting when the plate is close to the free surface. This is also when the numerical treatment becomes more difficult - the kernel is continuous for all $b>0$, but it is strongly singular if $b=0$. These facts lead us to consider the possibility of constructing an asymptotic approximation for small values of $b$. Some idea of the difficulties involved can be obtained by examining Hutson's 


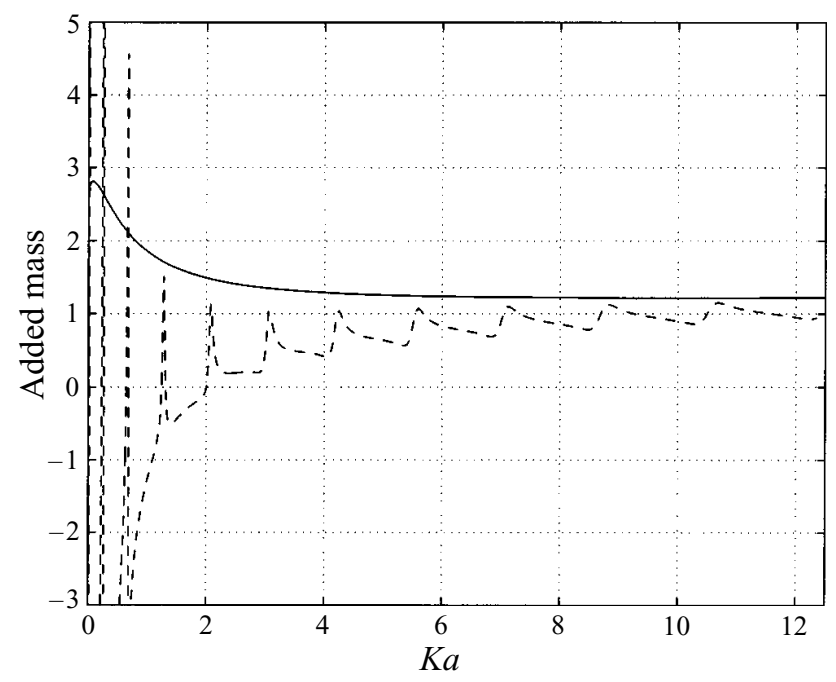

FIGURE 5. The added-mass coefficient for the submerged disc at $b=0.02$ (dashed curve) compared with the added-mass coefficient for the dock $(b=0$; solid curve). The difference between the two curves is approximately $\pi /(K a)$.

rigorous asymptotic analysis of Love's equation $(K=0$, so that all wave effects are absent) as $b \rightarrow 0$ (Hutson 1963). This work is ongoing, and will be described elsewhere.

The diffraction problem, where an incident regular wavetrain is scattered by a fixed disc, can be treated in a similar manner. In particular, the exciting force on the disc can be found by solving the simple integral equation (7.6), except that the function $x$ on the right-hand side must be replaced by $\sin K x$; see Appendix B.

This paper is dedicated to the memory of Gerry Wickham, a superb teacher, colleague and friend.

This paper was completed while the first author (P.A.M.) was on sabbatical leave in the Department of Mathematical and Computer Sciences, Colorado School of Mines. He thanks the Department for its kind hospitality, and acknowledges receipt of a Fulbright Scholarship Grant. The second author (L.F.) acknowledges financial support from CAPES (Brazil). Both authors thank an anonymous referee for offering the explanation given at the end of $\S 9$.

\section{Appendix A. Two-dimensional finite-part integrals}

The hypersingular integral in (3.5) can be defined in several equivalent ways. Assume, for simplicity, that $\Omega$ is a flat bounded region in the $(x, y)$-plane. Then, for a sufficiently smooth function $w$ (in fact, we require that $w$ has tangential derivatives which are themselves Hölder continuous), one natural definition in the context of boundary-value problems is

$$
f_{\Omega} w(\xi, \eta) \frac{\mathrm{d} \Omega}{R^{3}}=\lim _{z \rightarrow 0} \frac{\partial}{\partial z} \int_{\Omega} w(\xi, \eta)\left\{\lim _{\zeta \rightarrow 0} \frac{\partial}{\partial \zeta}\left(\frac{1}{\left[R^{2}+(z-\zeta)^{2}\right]^{1 / 2}}\right)\right\} \mathrm{d} \Omega,
$$


where $\mathrm{d} \Omega=\mathrm{d} \xi \mathrm{d} \eta$. Another definition is

$$
f_{\Omega} w(\xi, \eta) \frac{\mathrm{d} \Omega}{R^{3}}=\lim _{\varepsilon \rightarrow 0}\left\{\int_{\Omega \backslash \Omega_{\varepsilon}} w(\xi, \eta) \frac{\mathrm{d} \Omega}{R^{3}}-\frac{2 \pi w(x, y)}{\varepsilon}\right\},
$$

where $\Omega_{\varepsilon}$ is a small disc of radius $\varepsilon$ centred at the singular point $(x, y)$. Both of these definitions have analogues for one-dimensional finite-part integrals. For more information on hypersingular integrals over surfaces, see Martin \& Rizzo (1989, 1996), Krishnasamy et al. (1990) and Krishnasamy, Rizzo \& Rudolphi (1992).

\section{Appendix B. The diffraction problem}

A wave with surface elevation $A \cos (K x-\omega t)$ is scattered by a fixed horizontal submerged disc. Thus, the incident potential is

$$
\phi_{i n c}=-\mathrm{i}(g / \omega) A \mathrm{e}^{-K z} \mathrm{e}^{\mathrm{i} K x} .
$$

The boundary condition is (3.1), wherein $V=-\partial \phi_{i n c} / \partial n$. Expanding $V$ as (5.1) gives

$$
V_{n}(r)=-\frac{1}{4} \pi K D_{n} J_{n}(K r) \quad \text { with } \quad D_{n}=(4 / \pi)(g / \omega) A \mathrm{e}^{-K b / 2} \epsilon_{n} i^{n+1} .
$$

Hence, using (6.5), (6.3) gives $\psi_{n}^{\infty}(x)=K x j_{n}(K x)$.

The exciting force on the disc is given by (3.6), wherein $[\phi]$ is the discontinuity in the scattered potential $\phi$ across the disc. (Note that $\left[\phi_{i n c}\right]=0$, so that the Froude-Krylov force vanishes.) $[\phi]$ can be expanded using (5.3) and (6.1), where $\psi_{n}$ solves (6.6). However, the (vertical) exciting force is given completely in terms of $\psi_{0}$; it is

$$
8 \rho g a^{2} A \mathrm{e}^{-K b / 2} \operatorname{Re}\left\{\mathrm{e}^{-\mathrm{i} \omega t} \int_{0}^{1} \psi_{0}(x) x \mathrm{~d} x\right\} .
$$

Moreover, $\psi_{0}$ solves the simple integral equation (7.6), except that the forcing function on the right-hand side is now $K x j_{0}(K x)=\sin K x$ (rather than $x$ ).

\section{REFERENCES}

Alves, C. \& Ha Duong, T. 1995 Numerical experiments on the resonance poles associated to acoustic and elastic scattering by a plane crack. In Mathematical and Numerical Aspects of Wave Propagation (ed. G. Cohen, E. Bécache, P. Joly \& J. E. Roberts), pp. 544-553. SIAM, Philadelphia.

Atkinson, W. J., Young, J. H. \& Brezovich, I. A. 1983 An analytic solution for the potential due to a circular parallel plate capacitor. J. Phys. A: Math. Gen. 16, 2837-2841.

Collins, W. D. 1961 On the solution of some axisymmetric boundary value problems by means of integral equations V. Some scalar diffraction problems for circular disks. Q. J. Mech. Appl. Maths 14, 101-117.

FARINA, L. 1996 Interaction of water waves with thin circular plates. PhD thesis, University of Manchester.

Garrett, C. J. R. 1971 Wave forces on a circular dock. J. Fluid Mech. 46, 129-139.

Guidera, J. T. \& Lardner, R. W. 1975 Penny-shaped cracks. J. Elast. 5, 59-73.

HAZARD, C. \& LENOIR, M. 1993 Determination of scattering frequencies for an elastic floating body. SIAM J. Math. Anal. 24, 1458-1514.

Hughes, B. D. 1984 On the potential due to a circular parallel plate capacitor. J. Phys. A: Math. Gen. 17, 1385-1386.

Hutson, V. 1963 The circular plate condenser at small separations. Proc. Camb. Phil. Soc. 59, 211-224.

KIM, W. D. 1963 The pitching motion of a circular disk. J. Fluid Mech. 17, 607-629. 
Krishnasamy, G., Rizzo, F. J. \& RudolPhi, T. J. 1992 Continuity requirements for density functions in the boundary integral equation method. Computational Mech. 9, 267-284.

Krishnasamy, G., Schmerr, L. W., Rudolphi, T. J. \& Rizzo, F. J. 1990 Hypersingular boundary integral equations: some applications in acoustic and elastic wave scattering. J. Appl. Mech. 57, 404-414.

Lamb, H. 1932 Hydrodynamics, 6th edn. Cambridge University Press.

Linton C. M. \& Evans, D. V. 1992 The radiation and scattering of surface waves by a vertical circular cylinder in a channel. Phil. Trans. R. Soc. Lond. A 338, 325-357.

Linton C. M. \& Evans, D. V. 1993 Hydrodynamic characteristics of bodies in channels. J. Fluid Mech. 252, 647-666.

Longuet-Higgins, M. S. 1967 On the trapping of wave energy around islands. J. Fluid Mech. 29, 781-821.

Love, E. R. 1949 The electrostatic field of two equal circular co-axial conducting disks. Q. J. Mech. Appl. Maths 2, 428-451.

Love, E. R. 1990 The potential due to a circular parallel plate condenser. Mathematika 37, 217-231.

MaCCAMy, R. C. 1961 On the scattering of water waves by a circular disk. Arch. Rat. Mech. Anal. 8, 120-138.

MaedA, H. 1981 On the hydrodynamic forces for shallow draft ships in shallow water. Naval Arch. Ocean Engng. 19, 54-67.

Martin, P. A. \& Rizzo, F. J. 1989 On boundary integral equations for crack problems. Proc. R. Soc. Lond. A 421, 341-355.

Martin, P. A. \& Rizzo, F. J. 1996 Hypersingular integrals: how smooth must the density be? Intl J. Numer. Meth. Engng 39, 687-704.

Martin, P. A. \& Wickham, G. R. 1983 Diffraction of elastic waves by a penny-shaped crack: analytical and numerical results. Proc. R. Soc. Lond. A 390, 91-129.

McIver, P. \& Evans, D. V. 1984 The occurrence of negative added mass in free-surface problems involving submerged oscillating bodies. J. Engng Maths 18, 7-22.

MiLES, J. W. 1971 A note on variational principles for surface-wave scattering. J. Fluid Mech. 46, $141-149$.

MiLEs, J. W. 1986 Resonant amplification of gravity waves over a circular sill. J. Fluid Mech. 167, $169-179$

MiLes, J. W. 1987 On surface-wave forcing by a circular disk. J. Fluid Mech. 175, 97-108.

Miles, J. \& Gilbert, F. 1968 Scattering of gravity waves by a circular dock. J. Fluid Mech. 34, 783-793.

Newman, J. N. 1977 Marine Hydrodynamics. MIT Press.

Newman, J. N., Sortland, B. \& Vinje, T. 1984 Added mass and damping of rectangular bodies close to the free surface. J. Ship Res. 28, 219-225.

Parsons, N. F. \& Martin, P. A. 1992 Scattering of water waves by submerged plates using hypersingular integral equations. Appl. Ocean Res. 14, 313-321.

Parsons, N. F. \& Martin, P. A. 1994 Scattering of water waves by submerged curved plates and by surface-piercing flat plates. Appl. Ocean Res. 16, 129-139.

Parsons, N. F. \& Martin, P. A. 1995 Trapping of water waves by submerged plates using hypersingular integral equations. J. Fluid Mech. 284, 359-375.

Poisson, O. 1995 Étude numérique des pôles de résonance associés à la diffraction d'ondes acoustiques et élastiques par un obstacle en dimension 2. Math. Modelling \& Numer. Anal. 29, 819-855.

Sneddon, I. N. 1966 Mixed Boundary Value Problems in Potential Theory. North-Holland.

Tanaka, Y., Намамото, T. \& Наsнiмото, А. 1981 Free vibration of floating circular plates. Proc. 31st. Japan Natl Congress for Appl. Mech. 31, 289-302.

Yu, X. \& CHWANG, A. T. 1993 Analysis of wave scattering by submerged circular disk. J. Engng Mech. 119, 1804-1817.

Yu, Y. S. \& UrSell, F. 1961 Surface waves generated by an oscillating circular cylinder on shallow water: theory and experiment. J. Fluid Mech. 11, 529-551.

Zhang, S. \& Williams, A. N. 1996 Wave scattering by submerged elliptical disk. J. Waterway, Port, Coastal Ocean Engng 122, 38-45. 